\title{
El desarrollo de capacidades y la gestión del conocimiento en los potenciales de cambio y competitividad universitaria*
}

Recibido: 2015-02-20 -Enviado a pares: 2015-03-10

Aprobado por pares: 2015-04-05 Aceptado: 2015-04-30

\author{
Jan-Ove Christer Olsson* \\ Julio César Acevedo Tabares***
}

\section{Resumen}

El presente artículo se centra en la consideración de que hay empresas que desconocen los mecanismos necesarios para preservar y dinamizar los conocimientos que se generan en sus interacciones cotidianas a través de los roles y perfiles profesionales de quienes forman parte de ellas. Se parte del supuesto que estas empresas, por estar atentas a las cadenas de producción, no centran su mirada en la gestión del conocimiento, posibilitando la fuga del mismo hacia otros sectores, lo que constituye una pérdida irreparable respecto al potencial humano y al capital cognitivo. Con el objetivo de adquirir conocimiento sobre cómo se puede mejorar y desarrollar la capacidad profesional en lo que respecta al aprendizaje, las TIC y las estrategias de comunicación dentro de un marco de una organización competitiva se realizó el proyecto a partir de un estudio de caso en la Universidad de Medellín y su Departamento de Ciencias Básicas bajo una metodología cualitativa y usando el método etnográfico. Entre los resultados se puede resaltar: 1 . Si la gestión que implica evaluar el desempeño de los docentes no funciona bien, existe la posibilidad de que se sientan desmotivados para realizar cambios profesionales; 2. El proyecto Permanencia con Calidad muestra un proceso de cambio y con ello un desarrollo de capacidades profesionales y una nueva práctica, lo cual se manifiesta en la manera en que los docentes se expresan sobre su rol profesional.

Palabras clave: competencias comunicativas, comunicación para el desarrollo, comunicación y educación, capital cultural, formación continua.

Este artículo es el resultado de un proceso investigativo realizado en la Universidad de Medellín-Colombia, durante el periodo 2012-2013, en la convocatoria interna de proyectos. (Colombia)

.* Magíster de Ciencia en Educación con enfoque de aprendizaje, comunicación y tecnologías de información y comunicación. Investigador principal del proyecto actual y miembro del grupo Holográfico, adscrito a la Facultad de Comunicación de la Universidad de Medellín. Profesor de tiempo completo del programa Comunicación Gráfica Publicitaria. E-mail: jolsson@udem.edu.co

... Filósofo, magíster en Educación Superior. Miembro Investigador del grupo COP - Comunicación Organización y Política, adscrito a la Facultad de Comunicación de la Universidad de Medellín. Profesor tiempo completo de Epistemología de la Comunicación.E-mail: jcacevedo@udem.edu.co 


\title{
The development of capabilities and the management of knowledge in the potentials of university competitiveness and change
}

\begin{abstract}
This article is focused on the consideration that there are companies that do not know the mechanisms necessary for boosting the dynamism and preserving the knowledge generated in their daily interactions by means of the professional profiles and roles of the people that work for those companies. The study is based on the supposition that these companies, due to being focused on the chains of production, do not aim their attention at the management of the knowledge, allowing it to leak into other sectors. This knowledge leak constitutes an irreparable loss in terms of the human potential and cognitive capital. With the objective of acquiring knowledge on how to improve and develop the professional capability regarding the learning, the information and communication technologies and the communication strategies within a framework of a competitive organization, the project was carried out based on a case study in Universidad de Medellín and its Department of Basic Sciences under a qualitative methodology and using the ethnographic method. The following are several results worth highlighting: 1 . If the management necessary for evaluating the performance of the professors does not work adequately, there is a possibility that they feel unmotivated to undergo professional changes; 2. The "Permanencia con Calidad" (Remaining in the post with quality) project shows a process of change accompanied by the development of professional capabilities and a new practice, which is manifested in the way the professors express themselves about their professional role.
\end{abstract}

Key words: communicational competence, communication for development, communication and education, cultural capital, continuous training.

\section{O desenvolvimento das capacidades e a gestão do conhecimento nos potencias de mudança e competitividade universitária}

\section{Resumo}

O presente artigo se centra na consideração de há empresas que desconhecem os mecanismos necessários para manter e dinamizar os conhecimentos que se geram nas suas interações cotidianas por meio dos papéis e perfis profissionais daqueles que são parte delas. Baseia-se no pressuposto de que essas empresas por estar atentas a estas cadeias de produção, não centram sua atenção na gestão do conhecimento, permitindo a fuga dele para outros sectores, o que constitui uma perda irreparável para o potencial humano e ao capital cognitivo. A fim de adquirir conhecimento do come pode melhorar e desenvolver a capacidade profissional no que respeita à aprendizagem, as TIC e as estratégias de comunicação no âmbito de uma organização competitiva se realizou o projeto a partir de um estudo de caso na Universidade de Medellín e seu departamento de Ciências Básicas com base numa metodologia qualitativa e usando o método etnográfico. Entre os resultados se pode ressaltar: 1. Se a gestão que envolve avaliar o desempenho dos professores não funciona bem, é possível que eles se sentem desmotivados para fazer mudanças de carreira; 2. O projeto "Permanencia com Calidad" mostra um processo de mudança e com ela um desenvolvimento das capacidades profissionais e uma nova pratica, que se manifesta na maneira como os professores se expressam do seu papel profissional.

Palavras-Chave: habilidades de comunicação, comunicação de desenvolvimento, comunicação e educação, capital cultural, a educação continuada 


\section{Introducción}

Vivimos en un mundo caracterizado por una economía globalizada y un mercado de trabajo con una mayor tasa de cambio. En este ambiente, los profesionales enfrentan la necesidad de una constante reflexión y autocrítica sobre sus roles y desempeño, así como se les exige de igual modo a las organizaciones. El capital humano se ha vuelto un factor muy importante en la fuerte competencia y en el contexto de un mercado caracterizado cada vez más por la internacionalización. Desde las dos perspectivas, la de la organización y la del profesional, el desarrollo de las capacidades o competencias del recurso humano se vuelve un asunto altamente estratégico. Sobre esta temática se ha enfocado el presente proyecto de investigación que, a continuación, expone las principales observaciones y conclusiones en cuanto a procesos de cambio y a su importancia y potencial para el desarrollo de las competencias del docente universitario por medio del aprendizaje en el entorno laboral. El enfoque metodológico ha sido principalmente descriptivo y en la tradición etnográfica-cualitativa.

\section{Material y métodos. El marco teórico}

La relación con el campo de investigación del aprendizaje y la enseñanza en las economías del conocimiento se construye predominantemente sobre las bases teóricas de la cultura, el comportamiento y la estructura organizacionales, la inteligencia artificial, la gestión de calidad, la gestión estratégica, la economía de la información y la mediación del desempeño organizacional (Baskerville E Dulipovici, 2006: p. 83). Baskerville y Dulipovici argumentan que la gestión del conocimiento ha surgido como un campo importante de práctica e investigación en los sistemas de información. Las teorías en este contexto están utilizándose para proveer una base racional para la administración del conocimiento, para definir el proceso requerido para hacerlo, e identificar cómo se evalúan los resultados de dicho proceso.

La comunicación como parte de la gestión del conocimiento la describen Ming Mei, Ting Lee, y Al-Hawamdeh, (2004: p. 12) cuando buscan identificar una estrategia efectiva de comunicación en relación con la necesidad de implementar la gestión del conocimiento en la organización Ellos afirman que la estrategia de comunicación examinada en su estudio de caso de la Universidad del Servicio Público (Civil Service College) de Singapur facilita la comunicación efectiva entre el staff de manera que pueda ocurrir una eficaz generación y socialización del conocimiento. Los autores argumentan que el rol estratégico del conocimiento en el nivel organizacional ha alcanzado un elevado estatus de interés especialmente en lo referente al uso de "procesos de compartir conocimiento" con el propósito de capturar y almacenar el conocimiento de la organización. También señalan que la tecnología de información y comunicación ha creado una infraestructura para dirigir el conocimiento y para dinamizar las oportunidades de gestión del mismo. Aún más, hacen énfasis en que no es suficiente invertir en tecnología y en que la organización no puede descuidar o subestimar las cuestiones culturales en el ambiente organizacional, pues que juegan un papel importante en determinar el éxito general de las iniciativas de gestión del conocimiento (GC). Asimismo, afirman que la GC trata esencialmente del manejo del 
cambio y se refieren a estudios relativos a la cultura corporativa como factor crucial para el éxito de los esfuerzos de GC. Cuando se necesita hacer cambios en la organización, esto requiere nuevas formas de trabajar y de comportamiento, y un cambio en las normas culturales y las mentalidades de los empleados. El cambio tiene que ocurrir en el nivel individual y para lograr eso es crucial que la administración comunique apropiadamente por qué los cambios son metas importantes y deseables para la organización.

En tal sentido, la aseveración de Costa al establecer la intrincada relación entre comunicación, desempeño laboral e interacciones está en que ellas ingresan al dinámico campo de los ecosistemas de las actuaciones. "Es indiscutible que todas las acciones y actuaciones implican comunicación". Diferentes modos de actuación y de conductas constituyen un conjunto que permite separar artificialmente la comunicación de la actuación. Según el autor "lo que importa, más que el inventario, la frecuencia y la relevancia de estos y otros modos de comunicar y de actuar, es la coherencia que debe existir entre ambos universos (Costa, 1995: p 210). A su vez, esta coherencia es dada por la cultura y la ética empresarial.

Otra posición y enfoque interesante para el proyecto se centró en la función interpretativa (Saladrigas Medina, 2005). Esta posición enfatiza el papel de la construcción simbólica como el enfoque interpretativo-simbólico y las denominadas teorías posmodernas. Las matrices aquí se encuentran en la antropología social o cultural. Las concepciones del investigador Martín Barbero consideran la cultura como un factor importante para comprender la naturaleza comunicativa, es decir, su carácter de proceso productor de significaciones y no de mera circulación de informaciones, puesto que el receptor no es un simple descodificador de lo que puso el emisor en el mensaje, sino un productor también. Federico Varona afirma, según el mismo artículo, que la perspectiva interpretativa ve a las organizaciones como culturas, las cuales poseen un conjunto de creencias y valores y un lenguaje que se reflejan en "los símbolos, los ritos, las metáforas, las historietas, en el sistema de relaciones y en el contenido de las conversaciones" (Saladrigas M. 2005: p. 7).

Esta perspectiva interpretativa exige más de entendimiento que realizar cambios en las prácticas de comunicación de una organización. Por tanto, el presente proyecto asume este enfoque teórico como un referente de apoyo y de validez para las implicaciones que pudiesen emanar de la actividad investigativa.

La comunicación es el vehículo de la identidad y la cultura. J. Costa plantea que "la comunicación es el flujo vital por el que la empresa reúne y coordina sus partes constitutivas como organismo", es decir: la comunicación como vehículo de la cultura (ya que toda cultura existe y se extiende por la comunicación), y ella misma como elemento propagador de su identidad. La comunicación es el factor de integración de los individuos en la empresa, y de esta en su entorno social. (Costa. 1995: p. 206).

\section{La metodología}

Inicialmente planteamos la pregunta: ¿Qué caracteriza el proceso de desarrollo de capacidad y la elaboración de aprendizaje, las TIC y las estrategias de comunicación de 
los profesionales en estudio (profesionales en la Educación Superior)? A partir de este problema global, se configura un estado de arte sobre aprendizaje en entornos laborales, sobre comunicación y productividad, sobre la teoría de la actividad, sobre gestión de conocimiento y competencia, sobre sistemas de información y comunicación estratégica. Durante la segunda fase se aplica un estudio de caso en la Universidad de Medellín, más concretamente en el Departamento de Ciencias Básicas.

La intención inicial era la de encontrar el caso de estudio en el sector privado, lo que nos puso en contacto con cuatro empresas en la ciudad de Medellín. Todas las empresas mostraban un claro interés en la temática de la investigación y todas tenían necesidad de avanzar en sus actividades en cuanto a la gestión del recurso humano y la gestión del conocimiento; pero no sabían cómo hacerlo. No obstante, y a pesar del interés manifiesto, al final decidieron las empresas que no tenían posibilidad de participar y así nos encontramos sin el caso de estudio en el preámbulo de la investigación. Para no perder más tiempo se decide optar por un segundo camino, y preguntar a algunos jefes si se podía hacer la investigación en la Universidad de Medellín. Se quería tener una organización grande como caso, en el sentido de tener más de 100 empleados, que como organización ofreciera una infraestructura organizacional con políticas del desarrollo de capacidades y de gestión del recurso humano.

En la Universidad de Medellín, encontramos 290 profesores de tiempo completo, 1 profesor de medio tiempo y 513 profesores de cátedra, además de contar con 388 empleados no docentes. La muestra para este proyecto se centró en docentes e investigadores en la Educación Superior, tanto de cátedra como de tiempo completo. La selección de caso significa que como investigadores podíamos caer en el riesgo de ponderar la subjetividad y en el momento de análisis y conclusiones lo tuvimos en cuenta para buscar la distancia necesaria y no caer en la llamada ilusión de la transparencia. Por esta razón, conservamos una distancia en nuestra relación con los docentes y jefes en el Departamento de Ciencias Básicas y pensamos en ellos como objeto de estudio y no como colegas.

En cuanto a la recolección de datos, se hizo con la metodología empírico-analítica y la etnografía por medio de observaciones, encuestas, entrevistas y un grupo focal. Para la encuesta seleccionamos todos los profesores de tiempo completo (22) y 28 profesores de cátedra. Se contó con 33 participantes en la encuesta, lo que responde a 60 \% de los seleccionados, y de estos 33 hay 29 que aplicaron la encuesta, para un $58 \%$. La mayoría de los profesionales objeto de esta investigación se clasifica de la siguiente manera: 70 \% corresponde a hombres, y $30 \%$, a mujeres, indistintamente si eran catedráticos o de tiempo completo. Con un tiempo de permanencia o de vinculación laboral entre los siguientes parámetros: 51,52 \% han trabajado entre 0 y 5 años en la Universidad de Medellín, 12,12 $\%$ han trabajado entre 6 y 10 años, otro grupo tenemos en el rango 11-15 años (18,18\%) y más de 21 años (18,18\%) en la organización (tabla 1). 


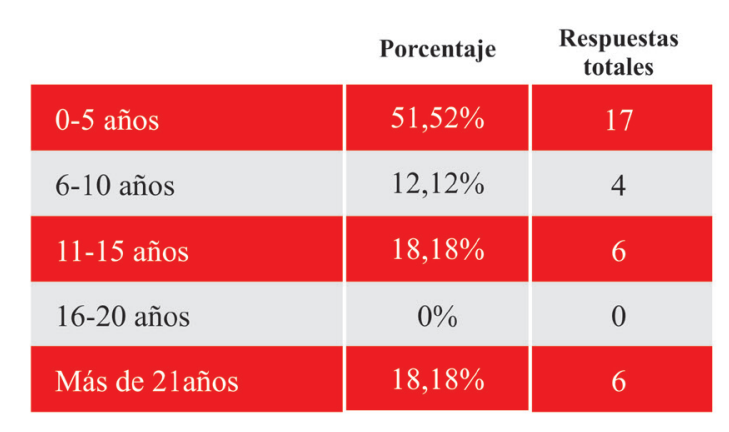

Tabla 1: Tiempo de vinculación laboral

Esto nos hace reflexionar sobre cierto grado de estabilidad laboral y de continuidad en la dinámica de los procesos y proyectos. El grupo más grande de profesores lleva entre 0-5 años en la institución, algo que evidencia que hay una gran entrada de recursos humanos con un posible impacto en cuanto a nuevas ideas y competencias. La universidad ha crecido mucho en los últimos 10 años, y la planta de profesores es más grande ahora para cubrir la demanda en la oferta de posgrados.

En cuanto la formación de los docentes se ha clasificado de la siguiente manera (tabla 2):

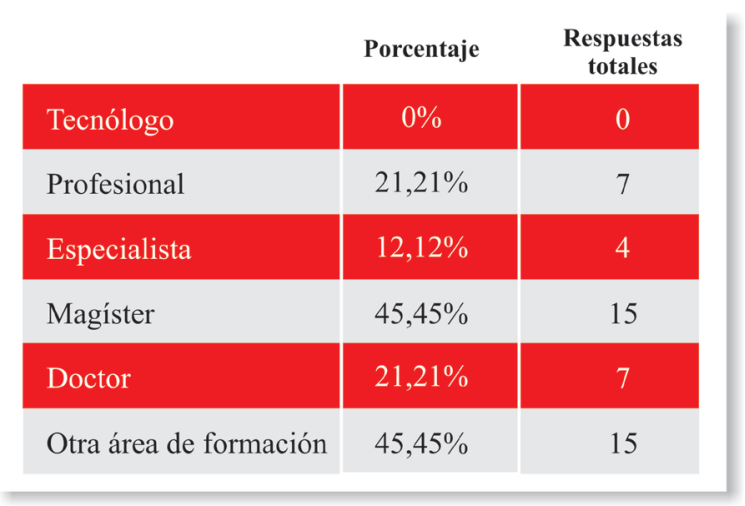

Tabla 2: El nivel de formación de los docentes e investigadores

La mayoría tiene alta formación como magíster o doctor (66,66 \%). Anteriormente, la Universidad contrataba profesores con Maestría pero ahora buscan profesores con doctorado, lo que fortalece la competitividad de la Institución. Las áreas de conocimiento de los profesionales en el estudio son: matemática, física, química, geofísica, ingeniería de materiales, ciencias básicas.

Para las entrevistas, seleccionamos seis profesores con contrato de tiempo completo. Entrevistamos a una profesora y a cinco a profesores, tres de ellos con un nivel de estu- 
dios de doctorado (Física, Química, Matemática) y otros con Maestría y Licenciatura en Matemáticas. Las entrevistas se grabaron en audio con sus respectivas transcripciones de audio a texto para el análisis y la interpretación. El alcance de estos audios es entre 16 y 44 minutos cada uno.

La dinámica con el grupo focal se centró en un conversatorio realizado con los líderes que, a consideración de los investigadores, tienen mayor responsabilidad en los procesos de gestión del conocimiento y del desarrollo de habilidades y competencias, como son: el jefe del departamento y la vicerrectora académica. Se realizó una grabación de vídeo con los dos investigadores y los líderes con una duración de 72 minutos, en la que se profundiza en algunos contenidos más incidentales de la investigación.

El proceso de análisis e interpretación de los datos se realizó mediante técnica de depuración de la información a través de referentes categoriales, en las siguientes etapas: la preparación y la descripción del material bruto, la reducción o depuración de datos, la aplicación de los métodos de análisis por contraste y el análisis transversal de los resultados encontrados con los diferentes instrumentos.

\section{Análisis y resultados}

Para desarrollar las capacidades o competencias en cada profesor e investigador es importante partir de un diagnóstico y análisis para identificar brechas, áreas débiles o áreas con oportunidad de mejoramiento, que pueden ser fundamentales a la hora de identificar las competencias centrales para el rol específico y sus funciones. Si la gestión que implica evaluar el desempeño de los docentes o profesionales no funciona bien, existe la posibilidad de que se sientan desmotivados para realizar cambios profesionales. Si los sistemas de evaluación y gestión funcionan bien y los docentes pueden capacitarse en áreas estratégicas, la organización gana ventajas competitivas con su capital humano. Una mejora de la gestión del recurso humano tiene que ver con la competencia del jefe inmediato; en este sentido, por los roles de su función es quien debe tener capacitación permanente.

\section{Descubrimientos, reflexiones y conclusiones sobre los problemas específicos de la investigación}

Sí, nosotros consideramos que existen factores específicos en la organización que afectan y controlan la forma como se estimula o retrasa un cambio en los profesionales para compartir sus conocimientos (sic) ${ }^{1}$.

Una de las necesidades más apremiantes que emergen del mundo laboral y profesional tiene que ver con los procesos de capacitación y actualización del personal al frente a las demandas que exigen las empresas, al verse exigidas por la incorporación de nuevos aprendizajes que las hagan más competitivas frente a las demás. Por eso las preguntas

Esta frase la tenemos en el reporte final del proyecto de investigación, p. 64, un reporte que fue evaluado por la Universidad de Medellín durante el mes diciembre 2013. 
cinco $^{2}$, seis ${ }^{3}$ y siete ${ }^{4}$ en la encuesta abordaron aspectos centrales para este proyecto investigativo sobre el desarrollo de las capacidades profesionales.

En cuanto a los mecanismos o vías que emplea la Universidad de Medellín para promover o propiciar la formación de sus empleados o profesionales y sus capacidades, las respuestas en la encuesta muestran una oferta de diferentes actividades como: apoyo a formación de alto nivel, diplomados, seminarios, maestrías, congresos, proyectos de investigación y cursos de especialización. La política de la institución es que el profesional, en nuestro caso docentes e investigadores, responden por media parte (o entre 50-60\%) y el empleador responde por la otra media parte del costo de la capacitación en varios casos.

Al indagar sobre las capacitaciones que ofrece la Universidad de Medellín, respecto a las necesidades de la misma, un $100 \%$ de los docentes se inclinan a favor del reconocimiento sobre la debida atención que asume la Universidad para con la formación y actualización de los mismos; dos aspectos que corroboran esta gestión se centran: el primero obedece a la tendencia de la Universidad por vincular o requerir mayor nivel de formación en docentes de tiempo completo como de cátedra, pues aún hay un 33 \% que requiere de Maestría y Doctorado; en segundo lugar, las indagaciones del proyecto arrojan que siendo satisfactoria la formación de los docentes, hay otras necesidades que requieren ser atendidas para potenciar dichos roles profesionales a favor de la organización, como por ejemplo las capacidades en cuanto: manejo de software, didáctica y habilidades comunicativas.

Cuando se indaga si los profesores participan en otras actividades de carácter formativo con el fin de desarrollar capacidades importantes para el ejercicio de su trabajo, las respuestas nos permiten constatar de manera directa que, aunque la universidad responda permanentemente por la capacitación y la actualización de sus docentes, muchos de ellos (87,5 \%) buscan por fuera de la universidad otros tipos de ofertas que les puedan ser útiles y pertinentes para optimizar su labor, como también deja en evidencia la necesidad que los docentes tienen de potenciar su perfil profesional en aras de otras ofertas de desempeño laboral.

Sobre la regularidad de actualizarse en el área de formación profesional y/o académica, indica que el ciclo de actualización es más corto en una sociedad construida sobre nuevas tecnologías y una economía de tipo globalizante. Los docentes y profesionales responden, en un 65,62 \%, que cada seis meses es necesario actualizarse, y en un 31,25 $\%$ responden que cada año es necesario actualizar su conocimiento.

¿Considera usted que la empresa -la Universidad de Medellín- favorece y apoya actividades o procesos de formación o capacitación de acuerdo a los perfiles laborales?

3 Enumere algunos mecanismos o vías que emplea la Universidad de Medellín para promover o propiciar la formación de sus empleados o profesionales y sus capacidades.

$4 \quad$ ¿Las capacitaciones que ofrece la Universidad de Medellín responden a las necesidades de la organización y/o la institución? 
Se reflexiona sobre posibles factores que pueden retrasar un cambio en los profesionales para desarrollar y compartir sus conocimientos, por ello es importante resaltar algunos factores: 1) el horario docente, 2) los costos para la capacitación, y 3) la gestión del desempeño. El horario normal es de 8:00 a. m. a 12:00 m. y de 3:00 p. m. a 7:00 p. $\mathrm{m}$. y si una capacitación o un evento se debe realizar después de cumplir una jornada completa existe el riesgo de sentirse desmotivado o cansado para iniciar este tipo de actividad cognitiva. En cuanto a si la gestión del desempeño no funciona bien, existe la posibilidad de tener profesionales que pierden sus incentivos para realizar cambios profesionales. Hay evidencia en las entrevistas y en la encuesta de que no se cuenta con el tiempo suficiente para realizar todas las actividades que un docente quisiera realizar, por lo que estiman conveniente priorizar actividades.

Otros factores que los profesores expresan como limitantes para el desarrollo y potenciación de las capacidades necesarias para un desempeño competitivo son:

- La falta de formación doctoral

- La falta de ejecución de proyectos de investigación internacionales

- El no fomento de las redes de investigación internacional

- La falta de escritura de artículos y libros de divulgación mundial en inglés

- La falta de integración de las funciones sustantivas de la universidad en todas las actividades que desarrolla el empleado

- La falta de vínculos con la empresa, el Estado y la sociedad

- La falta de participación en eventos nacionales e internacionales

- El lugar donde se desarrolla la capacitación

- Acceso a revistas de mayor impacto

- Actualización

- La falta de capacitaciones en los conocimientos de punta

- Por existir otros compromisos

- Falta de actitud

- Es necesario apoyar los procesos de comunicación entre los equipos de trabajo

- Demasiada carga académica y/o de investigación

- La falta de capacitación continua

El desarrollo de la investigación permitió identificar actividades y herramientas de desarrollo de las capacidades profesionales especialmente importantes para los profesores en su ambiente de trabajo, tales como: 
Apoyo a formación de alto nivel, diplomados, seminarios, congresos, foros, proyectos de investigación, maestrías y cursos de especialización. Herramientas como la plataforma U-virtual y tecnologías de información y comunicación como correo, computador, video beam, Internet, conexión wifi, wikis, chats, simuladores, bases de datos científicas, tableros inteligentes, cámaras, tablets, centro docente de cómputos, aulas adaptadas a talleres y consultorías y software especializado, por ejemplo Matlab y Cabri geometric para resolver problemas específicos 5 .

La situación laboral hoy en día es en alto grado dependiente de tecnologías y sistemas de información y comunicación. Esto da a entender que las dinámicas del ejercicio docente en las universidades han superado la postura tradicional de tiza y tablero y, en su efecto, son muchas las actividades académicas que se abordan con la presencia y soporte de las tecnologías, así como para la preparación de clases, las labores administrativas y en actividades de capacitación e investigación.

Sobre la periodicidad y de la importancia que tienen las distintas ayudas o apoyo de las herramientas tecnológicas, los datos de la investigación muestran la dinámica cotidiana que tiene la labor del docente en los procesos de construcción del conocimiento, así como de la transferencia del mismo en el aula y por fuera de ella.

En tal sentido, el recurrir a ellas como referencia funcional, diariamente, semanalmente o de manera quincenal, se vislumbra como un indicador que exige la institución para estar a la vanguardia sobre los usos de las herramientas tecnológicas. Esto dispone a los profesionales a incorporar sus discursos en el sentido más práctico posible, afianzando en los futuros profesionales el dominio por dicho recurso tecnológico.

Otro aspecto que resalta es el Seminario de Cualificación Docente como una actividad estratégica para el desarrollo de las capacidades que tienen los profesionales de dinamizar y contribuir al desempeño de sus roles profesionales entre ellos mismos. Esta actividad evidencia el apoyo y el acompañamiento de las áreas administrativas y coordinaciones académicas para fortalecer las relaciones y los resultados entre profesores, y entre profesores y estudiantes. Este tipo de actividades para los investigadores es particularmente incidental porque permite la confluencia y diálogo de saberes interdisciplinares no solo entre los docentes sino, probablemente, entre los programas que apoya constantemente con sus funciones.

Otro recurso significativo en el caso del Departamento de Ciencias Básicas tiene que ver con el proyecto Permanencia con calidad. A partir de la descripción entendemos que este proyecto:

Pretende reducir los fenómenos de deserción y pérdida académica, interviniendo la problemática desde cuatro categorías: la categoría académica, la categoría económica, la categoría personal y la categoría de adaptación a la cultura universitaria, que se desarrolla

Estas respuestas las hemos identificado a partir de las respuestas de nuestra encuesta realizada durante el proyecto de investigación. 
y apoya estratégicamente con diversos programas de carácter académico y de bienestar estudiantil (Universidad de Medellín, 2012).

Con un problema concreto a solucionar planearon acciones y empezaron un significativo proceso de cambio. Una decisión estratégica, como la creación del proyecto Permanencia con calidad, significa que la organización inicia un proceso de cambio y en procesos de cambio existe una dinámica y fuerza donde hay aprendizaje, o donde aparece aprendizaje (Olsson, 2008: pp. 5 y 75).

Mediante la aplicación de un modelo con una perspectiva triangular para el análisis sobre cómo la organización puede apoyar la práctica y el proceso cultural que lo produce argumenta la necesidad de buscar el equilibrio óptimo en la relación: Práctica (Practice), Tecnología de Información y Comunicación (Information and Communcation Technology, ICT), y Aprendizaje + Procesos de Cambio (Learning + Change Processes) (Olsson, 2008, p. 5 , p. 75, p. 90$)$.

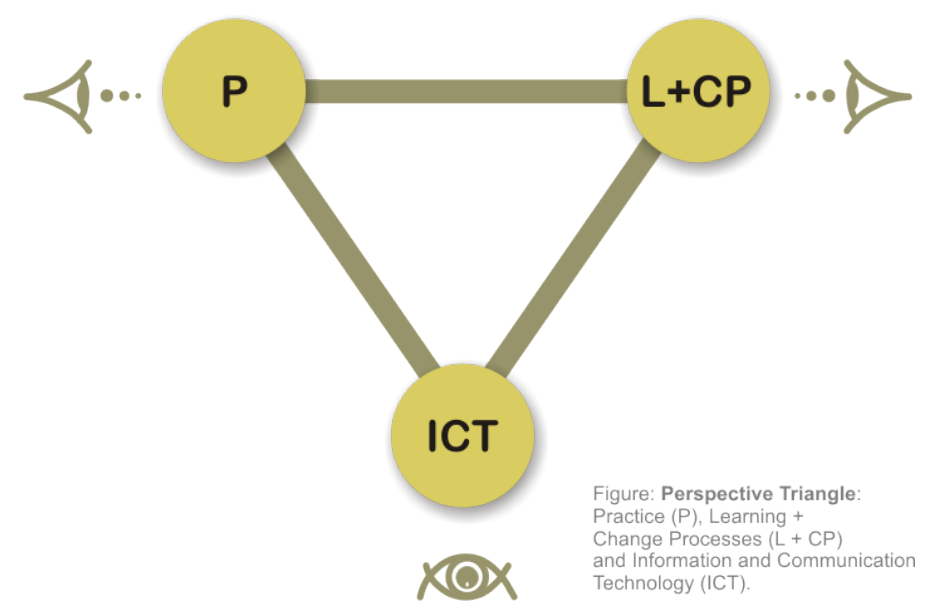

Gráfica: Perspective Triangle (Olsson, 2008)

Una práctica tiene la posibilidad de desarrollar un currículo de aprendizaje ("learning curriculum") (Lave $\mathcal{E}$ Wenger, 1991, p. 97) o un currículo de enseñanza ("teaching curriculum"), donde la primera se trata de la posibilidad de, en una manera improvisada, desarrollar nueva práctica y la segunda se trata de una perspectiva con un punto de vista de enseñar algo a alguien.

A learning curriculum consists of situated opportunities to (thus including exemplars of various sorts often thought of as 'goals') for the improvisational development of new practice (Lave 1989). A learning curriculum is a field of learning resources in everyday practice viewed from the perspective of learners. A teaching curriculum, by contrast, is constructed for the instruction of newcomers. (Lave \& Wenger, 1991: p. 97)."

Con el proyecto Permanencia con calidad el Departamento de Ciencias Básicas ha evolucionado a una nueva práctica; podemos decir, una práctica con un currículo de 
aprendizaje donde la pregunta principal para los docentes puede ser: ¿̇e qué manera aprenden los estudiantes mejor y qué significa eso para el rol del profesor en general y para un profesor de ciencias básicas en particular? Los procesos de cambio pueden generar nuevos pensamientos, maneras de trabajar, maneras de expresarse, relaciones, redes, y la creación de nuevas identidades en los profesores (Olsson, 2008: p 75).

Los profesores en el Departamento de Ciencias Básicas han participado en un proyecto y un proceso de cambio que ha significado un desarrollo de sus capacidades profesionales y su manera de pensar sobre su rol profesional. Las entrevistas con los profesores reflejan sus distintas visiones o percepciones sobre el rol docente. En este sentido, un proyecto con estas características es una excelente forma de desarrollar capacidades profesionales, y si logran el objetivo de reducir la deserción con nuevas metodologías didácticas, los profesores pueden compartir sus conocimientos con nuevos colegas y con otros profesores de ciencias básicas o áreas afines en otras universidades, una tarea que los profesores ya han empezado.

Otros ejemplos de actividades en las que los docentes y profesionales reconocen ser fundamentales para la promoción de la transferencia del conocimiento, y que tienen gran impacto en el ejercicio de sus funciones como profesor e investigador son:

- Investigación articulada a la docencia

- Resultados de prácticas en laboratorios

- Uso de simulaciones

- Realización de congresos o seminarios con soporte investigativo

- La realimentación que ha exigido el proceso de re-acreditación

- En los comités de currículo y de evaluación, momento especial para compartir las experiencias de los docentes

- En la participación de seminarios

- Mediante el aprendizaje colaborativo con los pares académicos

- Con la utilización de las tecnologías de información y comunicación

- Las asesorías o consultorías que se ofrecen en la Universidad y por fuera de ella

- A través del programa "Aprender es una nota"

- A través de la rueda de negocios de la corporación Tecnnova

En cuanto a la influencia que los convenios, asesorías e investigaciones que se han realizado en colaboración con otras empresas, organizaciones o instituciones, y si Estas actividades favorecen el aprendizaje y el desarrollo de nuevo conocimiento y potencian nuevas capacidades profesionales, los profesores reconocen un gran impacto, no solo para el sector industrial, sino también para las actividades investigativas de la univer- 
sidad. El $100 \%$ de los que han intervenido en este proyecto reconocen que el diálogo con la demanda social en los distintos sectores empresariales incide notablemente para impactar las prácticas en la transferencia del conocimiento que se realizan dentro de la universidad, y que potencian hacia nuevas capacidades profesionales que demandan los sectores productivos.

Resulta especialmente significativo que los docentes reconozcan que el diálogo con pares académicos de otras instituciones los ubica en una perspectiva de mayor competitividad y liderazgo, toda vez que ello les exige ajustar planes y proyectos para mejores y eficaces resultados. Además, porque los distintos sectores productivos siempre van a exigir que sus profesionales estén a la vanguardia de la escala mundial productiva; esto en lo que respecta al conocimiento así como también, en lo que tiene que ver con el uso adecuado de las herramientas y equipos de producción.

Asimismo, insisten en el necesario apoyo de los procesos de comunicación entre los equipos de trabajo, ya que ello permite el contacto con los colegas y la posibilidad de discutir con ellos diferentes temáticas que ayudan enormemente para el fortalecimiento de la actividad académica e investigativa en la Universidad.

Paralelamente, otro de los aspectos que resaltan los profesores recae sobre la necesidad de fortalecer una dinámica de comunicación proactiva para fomentar o estimular el desarrollo de capacidades profesionales entre sus compañeros; esto incide con la cultura organizacional que caracteriza e identifica la Universidad, su filosofía institucional y sus valores corporativos. La Institución y su proyecto educativo exigen no solo idoneidad de parte del docente sino también actitud. No sería prudente que la Universidad respondiera a todas las necesidades y peticiones de los docentes, si estos a su vez no asumen compromiso y actitud de entrega para ella, que es donde radica, en parte, el sentido de pertenencia.

En dicho contexto, se puede ver la importancia de tener una buena implementación del sistema de gestión del talento o recurso humano para optimizar el potencial de los profesionales y la organización.

\section{Discusión y conclusiones}

La pregunta principal para desarrollar la competitividad puede ser: ¿de qué manera debe trabajar la organización para desarrollar su conocimiento y las capacidades de sus profesionales? No existen respuestas sencillas a esta pregunta porque las situaciones de las organizaciones y su recurso humano son diferentes en muchos sentidos y hay una combinación de elementos que puede ser muy diferente o muy similar. Lo que la organización puede hacer es identificar sus actividades especiales y sus conocimientos importantes y tratar de organizar la forma de crear, guardar, compartir, acceder, aplicar y evaluar su conocimiento de valor para las actividades esenciales y especiales.

El fin de la gestión del conocimiento es el de mejorar las prestaciones organizativas y se puede hacer a través de capacitación de los profesionales para crear, capturar, compartir 
y aplicar sus conocimientos colectivos para tomar decisiones óptimas (Moral, Suárez et al., 2007: p. 15). Si la organización es exitosa con su gestión del conocimiento, se puede lograr el objetivo general, que consiste en llegar a maximizar la efectividad y el retorno de la empresa, y allí relacionamos todo con los conocimientos, y con la manera y habilidad de renovarlos constantemente.

En tal sentido, la potenciación del talento humano se asocia cada vez más a las dinámicas comunicativas y de interacción en el interior de las corporaciones o empresas como factor de competitividad para las organizaciones, pues cuando el profesional o trabajador se encuentra en una situación cómoda y en un entorno amistoso, es más empático, está más receptivo y se comunica mejor. Ello induce a pensar que para explicar cosas complejas o difíciles de entender, lo mejor es tener a nuestro interlocutor delante para poder ver sus reacciones y asegurarnos de que entiende el mensaje.

Si la organización tiene múltiples sistemas para la evaluación y seguimiento del desempeño de los profesores e investigadores, como en nuestro caso, es importante coordinar el seguimiento y el análisis de los instrumentos que los dos sistemas ofrecen.

Respecto a los profesores de cátedra, la investigación muestra que la situación es diferente, comparado con los docentes de tiempo completo, en el sentido que la formación no es tanto al nivel de Doctorado (hay dos profesores con esta formación), es más al nivel de Maestría (36), Especialización (17) y como profesional (25). Aquí hacemos énfasis en la necesidad de fortalecer el potencial para profundizar en el saber específico. Lo potencial para desarrollar capacidades profesionales en cuanto la didáctica específica puede ser más grande también, teniendo en cuenta las diferencias de competencia entre profesores de tiempo completo y cátedra, aunque la investigación no tuvo como propósito indagar sobre cuántos profesores de cátedra han hecho los diplomados ofrecidos (por ejemplo de didáctica) por la Universidad como capacitación docente.

Los resultados muestran que muchos de los docentes insisten en el necesario apoyo de los procesos de comunicación entre los equipos de trabajo, ya que ello permite el contacto con los colegas y la posibilidad de discutir con ellos diferentes temáticas que ayudan enormemente para el fortalecimiento de la actividad académica e investigativa en la universidad. La competencia en comunicación puede ser un área de mejoramiento profesional, acompañado con el recurso tiempo laboral.

Otro factor a considerar se identifica tras las observaciones, en el sentido de que los profesores de cátedra no trabajan tanto en las oficinas de los profesores, de tal modo que están menos expuestos a las conversaciones informales y diálogos entre pares, que es una parte de la transferencia de cultura institucional y del conocimiento. En estos encuentros informales existe la posibilidad de que un conocimiento tácito se vuelva explícito, (Nonaka, E Takeuchi, 1995: p. 495).

Un proyecto como el de Permanencia con calidad, que el Departamento de Ciencias Básicas en la Universidad de Medellín ha incorporado a partir de una investigación del año 2004, significa un proceso de cambio y en procesos de cambio existe una dinámica donde 
el aprendizaje y la transferencia de conocimientos se pueden fortalecer en gran medida entre docentes e investigadores. Los docentes en el Departamento de Ciencias Básicas han participado en un proyecto y un proceso de cambio que ha significado un desarrollo de sus capacidades profesionales y su manera de pensar sobre su rol profesional. Esta experiencia, consideramos, debe ser compartida y difundida en la organización como un buen ejemplo.

Las entrevistas con los docentes evidencian características en su forma de expresarse sobre su nuevo rol docente, contrastando con una imagen un poco estereotipada de un profesor de ciencias básicas transmitiendo su conocimiento frente a los estudiantes. En este sentido, un proyecto de cambio con estas características es una excelente forma de desarrollar capacidades profesionales y si logran el objetivo de reducir la deserción con nuevas metodologías, bien vale la pena compartirla con otros establecimientos de Educación Superior del entorno.

\section{Referencias}

Baskerville, R. E Dulipovici, A. (2006). The theoretical foundations of knowledge management, Knowledge Management Research \& Practice, (4): 83-105.

Cornelissen, J. (2008). Corporate communication. A guide to theory and practice. London: Sage Publications.

Costa, J. (1995). Comunicación corporativa y revolución de los servicios. Madrid: Ediciones de las ciencias sociales.

Lave, J. \& Wenger, E. (1991). Situated learning - Legitimate peripheral participation. Cambridge, MA: Cambridge University Press.

Ming Mei, Y., Ting Lee, S. \& Al-Hawamdeh, S. (2004). Formulating a communication strategy for effective knowledge sharing. Journal of Information Science, 30 (1), pp. 12-22. DOI: 10.1177/0165551504041674.

Moral, A., Del Pazos, J., Rodríguez, E., Rodríguez Paton, A. E Suarez, S. (2007). Gestión del conocimiento. Madrid: Thomson Ed.

Nonaka, I. E Takeuchi, H. (1995). The knowledge-Creating Company: How Japanese Companies Create the Dynamics of Innovation. New York: Oxford University Press.

Olsson, J.O. (2008). Digital Medellín - communities of practice and ICT for organizational development, ISSN: 1651-4769. Gothenburg: University of Gothenburg.

Saladrigas Medina, H. (2005). Organizational communication: theoretical matrices and communicative focus. Revista Latina de Comunicación Social 60 enero - diciembre de 2005, La Laguna (Tenerife). Recuperado de internet el 15 de septiembre de 2013, de URL: http://www.ull.es/publicaciones/ latina/200540salabrigas.pdf

Universidad de Medellín (2012). Permanencia con calidad. Recuperado de Internet el 15 de noviembre de 2012, de URL: http://www.udem.edu.co/index.php/estudiantes-permanencia-con-calidad/ informacion-general-permanencia-con-calidad 